Onkologe 2012 · 18:658-659

DOI 10.1007/s00761-012-2310-2

Online publiziert: 4. August 2012

(c) Springer-Verlag 2012

G.G. Grabenbauer ${ }^{1} \cdot$ N. Senninger ${ }^{2}$

${ }^{1}$ Radioonkologie \& Strahlentherapie, Klinikum Coburg

${ }^{2}$ Klinik für Allgemein- und Viszeralchirurgie, WWU/UKM, Münster

\title{
Analkarzinom - im Westen nicht Neues?
}

Weise rundet die Betrachtung der Möglichkeiten systemischer Therapieoptionen im Falle der Fernmetastasierung das Heft ab. Schulte und Hofheinz (Mannheim) betonen, dass es neben der Standardchemotherapie mit Fluorouracil/Platin auch Daten zum Einsatz von Cetuximab gibt.

Bereits seit mehr als einem Jahrzehnt gilt die mit 2 Zyklen Mitomycin C und Fluorouracil kombinierte, simultane Radiochemotherapie als etablierte Primärbehandlung. Dabei konnte in randomisierten Studien weder der neoadjuante noch der adjuvante Einsatz einer platinbasierten Polychemotherapie die relevanten onkologischen Endpunkte verbessern. Klinisch erscheint es daher wesentlicher, Unterbrechungen der Radiochemotherapie zu vermeiden, da hierdurch die akzelerierte Repopulierung von Tumorzellen und konsekutiv die Rezidivwahrscheinlichkeit befördert wird. Darüber hinaus steht heute mit der intensitätsmodulierten Radiotherapie (IMRT) eine Technik zur Verfügung, die einerseits die akuten Nebenwirkungen an Risikoorganen wie Dünndarm und Blase deutlich senkt, andererseits aber auch erlaubt, eine effektive lokale Dosis von 50-59 Gy zu applizieren.

Zusammenfassend bleibt festzuhalten, dass das Analkarzinom biologische, pathologisch-anatomische und klinische Besonderheiten aufweist, die für eine Expertise in Diagnostik und Therapie essenziell sind. In jedem Fall ist die vertrauensvolle interdisziplinäre Zusammenarbeit die Basis zur erfolgreichen Therapie.

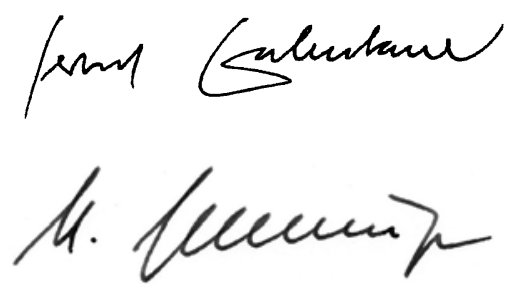

G.G. Grabenbauer und N. Senninger Für die Herausgeber des Leitthemas
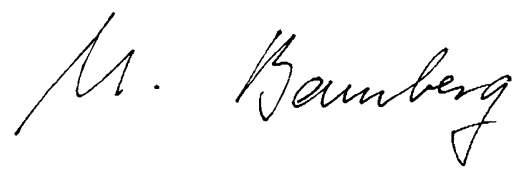

M. Bamberg

Für die Herausgeber

\section{Korrespondenzadressen}

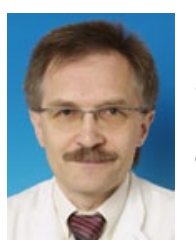

Prof. Dr. G.G. Grabenbauer Radioonkologie \& Strahlentherapie, Klinikum Coburg Ketschendorferstraße 33 96450 Coburg gg@strahlentherapie-coburg.de

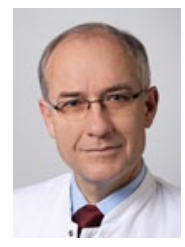

Prof. Dr. Dr. h.c. N. Senninger Klinik für Allgemeinund Viszeralchirurgie WWU/UKM Albert-Schweitzer-Campus 1, 48149 Münster senninger@uni-muenster.de 
Hier steht eine Anzeige.

黛 Springer 